\title{
Active Cognitive Lifestyle Associates with Cognitive Recovery and a Reduced Risk of Cognitive Decline
}

\author{
Riccardo E. Marioni ${ }^{\mathrm{a}, *}$, Ardo van den Hout ${ }^{\mathrm{b}}$, Michael J. Valenzuela ${ }^{\mathrm{c}, \mathrm{d}, \mathrm{e}}$, Carol Brayne ${ }^{\mathrm{a}}$, \\ Fiona E. Matthews ${ }^{\mathrm{b}}$ and MRC Cognitive Function and Ageing Study \\ ${ }^{a}$ Department of Public Health and Primary Care, University of Cambridge, Cambridge, UK \\ ${ }^{\mathrm{b}}$ MRC Biostatistics Unit, Cambridge, UK \\ ${ }^{\mathrm{c} S c h o o l ~ o f ~ P s y c h i a t r y, ~ U n i v e r s i t y ~ o f ~ N e w ~ S o u t h ~ W a l e s, ~ S y d n e y, ~ A u s t r a l i a ~}$ \\ ${ }^{\mathrm{d}}$ Brain and Ageing Research Program, Faculty of Medicine, University of New South Wales, Sydney, Australia \\ ${ }^{\mathrm{e}}$ Regenerative Neuroscience Group, Faculty of Medicine, University of New South Wales, Sydney, Australia
}

Handling Associate Editor: Sindre Rolstad

Accepted 28 August 2011

\begin{abstract}
Education and lifestyle factors linked with complex mental activity are thought to affect the progression of cognitive decline. Collectively, these factors can be combined to create a cognitive reserve or cognitive lifestyle score. This study tested the association between cognitive lifestyle score and cognitive change in a population-based cohort of older persons from five sites across England and Wales. Data came from 13,004 participants of the Medical Research Council Cognitive Function and Ageing Study who were aged 65 years and over. Cognition was assessed at multiple waves over 16 years using the Mini-Mental State Examination. Subjects were grouped into four cognitive states (no impairment, slight impairment, moderate impairment, severe impairment) and cognitive lifestyle score was assessed as a composite measure of education, mid-life occupation, and current social engagement. A multi-state model was used to test the effect of cognitive lifestyle score on cognitive transitions. Hazard ratios for cognitive lifestyle score showed significant differences between those in the upper compared to the lower tertile with a more active cognitive lifestyle associating with: a decreased risk of moving from no to slight impairment $(0.58,95 \% \mathrm{CI}$ $(0.45,0.74))$; recovery from a slightly impaired state back to a non-impaired state $(2.93(1.35,6.38))$; but an increased mortality risk from a severely impaired state $(1.28(1.12,1.45))$. An active cognitive lifestyle is associated with a more favorable cognitive trajectory in older persons. Future studies would ideally incorporate neuroradiological and neuropathological data to determine if there is causal evidence for these associations.
\end{abstract}

Keywords: All epidemiology, cognitive aging, cognitive reserve, education

\section{INTRODUCTION}

There is great interest on the impact of potentially modifiable factors such as education and lifestyle

*Correspondence to: Riccardo E. Marioni, Institute of Public Health, University Forvie Site, Robinson Way, Cambridge CB2 0SR, UK. Tel.: +44 (0)1223 763837; Fax: +44 (0)1223 330330; E-mail: rem50@medschl.cam.ac.uk. upon the progression of cognitive decline and the development of dementia. Evidence from large-scale epidemiological studies indicates that higher levels of education, occupational complexity, and cognitive leisure activities reduce the risk of incident dementia and cognitive decline [1,2]. A summary odds ratio from a review of 22 papers up to the end of 2004 found the effects of education, occupation, pre-morbid IQ, and mental activities reduce the risk of incident 
dementia by $46 \%$ [2]. It is possible that these factors provide functional protection from neuropathology, although the biological mechanisms underlying such processes are unclear. Two proposed mechanisms include disease modification and compensation. The former suggests a decreased risk for developing pathology while the latter indicates greater ability to cope with underlying damage. This overall protection from dementia and cognitive dysfunction can be referred to as 'cognitive reserve'. Stern [3,4] suggests factors such as brain size and neural networks are effective measures of a passive model (brain reserve), with childhood IQ, educational attainment, leisure activity, degree of literacy, and adult occupation being good markers of an active model (cognitive reserve).

Stern also presents evidence for higher reserve resulting in a poorer outcome once an individual has reached a dementia state [4]. Prospective evidence is cited for associations between increased education or occupational attainment and a faster transition to death in 246 patients with Alzheimer's disease (AD) [5]; the relative risk of mortality for those with $>8$ years of education was $1.76,95 \%$ CI $(1.11,2.77)$. In addition, a univariate analysis on 438 incident dementia cases found that those with more education had a reduced survival time, although this did not reach conventional significance [6]. However, such associations have not yet been tested in population-based cohorts of older persons prior to the onset of dementia. It may be possible that those with higher reserve have higher initial cognitive scores and a later onset of decline. This implies that by the time they reach an incident dementia state they have an extensive burden of underlying neuropathology and so a fast transition to death becomes more likely. On the contrary, those with lower reserve would be more likely to decline gradually and spend a longer time period with dementia. Such a model implies different slopes of decline for different individuals.

Previous studies have not investigated the association between reserve or cognitive lifestyle factors and transitions from normal cognition, through slight impairment to severe impairment and the transitions from all possible cognitive states to death. The aim of this paper was to study the association between cognitive lifestyle (assessed as a weighted measure of education, occupation, and current social engagement) and cognitive change in a population-based cohort of over 13,000 older persons from five sites across England and Wales. Cognitive change was analyzed using a multi-state modeling framework. Multi-state modeling has many advantages over other methods. For example, death and cognitive decline can be modeled explicitly within the same model; a joint model would be required in a continuous approach. Covariate effects on the transitions are also allowed to vary by transition; this would require a spline-type model in a continuous framework. Cut-points for the start and end of the spline sections would also have to be defined and these would be as arbitrary as the choices for the state based model. Furthermore, a three point change in a Mini-Metal Status Examination (MMSE) score can have different interpretations depending on the initial MMSE score. Analyzing transitions between established MMSE categories overcomes this problem. Back transitions or cognitive recovery can also be modeled with ease. While it is possible that an individual can be assigned to a group that does not reflect their true cognitive state, multi-state modeling also takes this into account by defining a hidden Markov model, which includes misclassification estimates.

\section{MATERIALS AND METHODS}

\section{Study population}

Data came from the Medical Research Council Cognitive Function and Ageing Study (MRC CFAS) [7]. MRC CFAS is a multi-center study on over 18,000 persons from across six centers in England and Wales; five of the centers have standardized designs. Only data from the five centers were considered for this analysis. These five centers had a two-phase sampling design with a screening interview followed by an assessment interview. Participants were selected from Family Health Service Authority lists, and were stratified by age to include persons aged 65 years and over at the index date for each center and living within a specified geographical area. The first meetings with the participants took place between 1989 and 1993. Follow-up cognitive testing was administered over a 16 year period with up to ten cognitive interviews in total. The times between assessments were similar for those within the screen and assessment arms but not between arms. For further details of the study design please see the CFAS website (http://www.cfas.ac.uk).

In this study, data were used from the five centers with a standardized design: Cambridgeshire $(n=$ $2,601)$, Gwynedd $(n=2,625)$, Newcastle $(n=2,524)$, Nottingham $(n=2,514)$, and Oxford $(n=2,740)$. The total sample size was 13,004 . Persons were excluded from the analysis if they: 1) only had a single data point, i.e., no transitions were recorded $(n=159)$; 2$)$ had baseline missing data that prevented calculation of a 
cognitive lifestyle score $(n=305)$; or 3 ) had a missing state at baseline $(n=246)$. This left a sample of 12,492 available for analysis of whom 5,032 (40.3\%) were men.

\section{Cognitive assessment}

Cognitive ability was assessed using the MMSE [8], which is a brief, easily administered measure of general cognitive function. This was assessed at multiple times in CFAS and was used to assign the subjects' cognitive states. The cognitive states were defined as follows: no cognitive impairment (MMSE 27-30); slight cognitive impairment (23-26); moderate cognitive impairment (18-22); severe cognitive impairment $(<18)$. The groupings were based around the slight cognitive impairment category, which is based on a figure from Stephan et al. [9]. They showed that, within a population representative sample, the MMSE is as effective a predictor of dementia risk as more complex measures of mild cognitive impairment (MCI). Figure 1 from the paper shows a score between 23 and 26 to clearly represent a mildly impaired group. The number of cognitive assessments an individual completed over the 16 year follow-up period ranged between one and ten: one test $(n=3,073,25 \%)$; two tests $(n=3,690,30 \%)$; three tests $(n=3,149,25 \%)$; four tests $(n=1,395,11 \%)$; five tests $(n=659,5 \%)$; six tests $(n=325,3 \%)$; seven tests $(n=64,<1 \%)$; eight tests $(n=44,<1 \%)$; nine tests $(n=56,<1 \%)$; ten tests $(n=37,<1 \%)$.

\section{Cognitive lifestyle score}

Cognitive lifestyle score (CLS) was defined using a criteria covering intensity of educational, occupational, and cognitive lifestyle activities in three phases of life (young adulthood, midlife, and late life) as previously shown to independently predict dementia incidence [10]. CLS correlates highly with the total score from the Lifetime of Experiences Questionnaire, for which it is a proxy measure [10]. It assimilates information about education, occupation classification, and current social engagement using a weighting system to enable all life stages to have the same median subscore. Education level in young adulthood was assessed by the self report question "how many years of full-time education?". Occupational complexity in midlife was assessed by recording the participant's main occupation in terms of years most worked and then recoding it using two systems, their social class grouping (from I to VI), and their socio-economic grouping (from 11 to 150). Social engagement (current levels in later life) was calculated on the basis of three 3-point Likert scale questions (i.e., min. 3 and max. 9) on contact with relatives and neighbors, and attending meetings. The types of meetings included community, church or social groups, such as over $60 \mathrm{~s}$ clubs, evening classes or other similar activities. Following common practice, gender-specific tertiles for the cognitive lifestyle scores were generated to investigate contrasting high, medium, and low cognitive lifestyle groups.

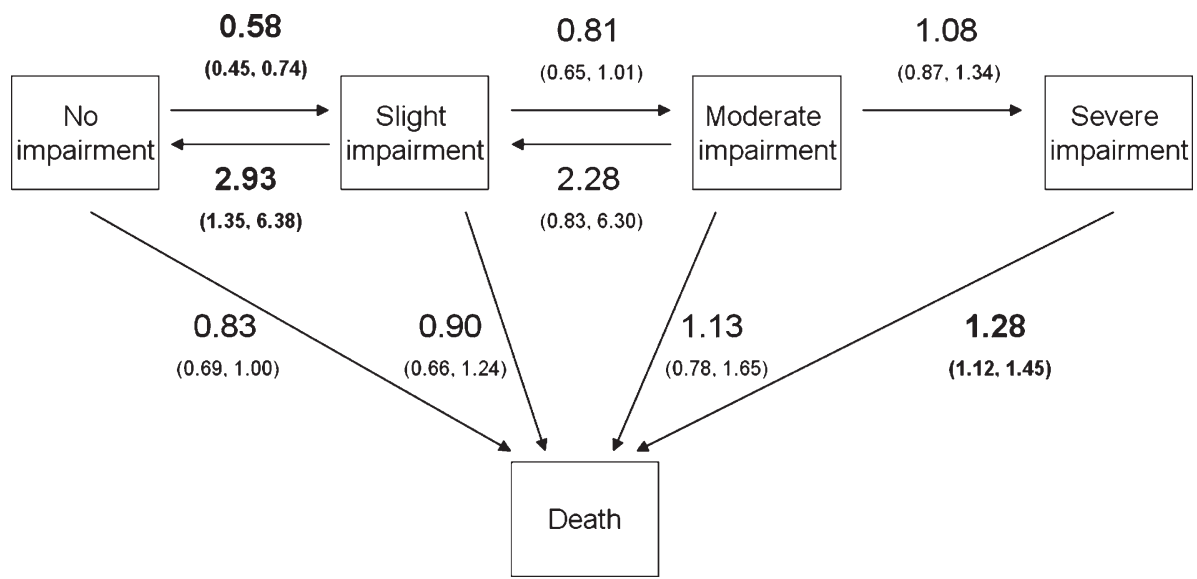

Fig. 1. Hazard ratios for cognitive state transitions - upper versus lower tertile of cognitive lifestyle. Over time an active cognitive lifestyle decreases chances of moving from normal to slightly impaired cognitive range (MMSE 23-26), and also increases chances of moving from slightly impaired to normal cognition by more than a factor of three. For individuals with major cognitive impairment $($ MMSE $<18)$, an active cognitive lifestyle increases chances of death by $28 \%$. 


\section{Statistical analysis}

A multi-state model was used to chart the progression of cognitive change. A four-state model was developed with death as an absorbing fifth state. Persons who were still alive at the end of the study were accounted for by right censoring. The probability of being in a particular state at a certain interview is conditional on the state occupied at the previous wave but not any states prior to this point. However, the model is not explicitly Markovian as the transition intensities are related to age, which is a time-dependent covariate. Neither is it a semi-Markov model as the time since entry into the state is not accounted for. To include the time to reach each state is complex as the exact times of state entry are unknown (interval censoring). Transitions were allowed between all cognitive states and death and between adjacent cognitive states with the exception of movement from the severely impaired state back to a moderately impaired state. Cognitive recovery from a severely impaired state is unlikely and there were insufficient data to model the transition. There is also potential for error in measuring cognitive ability, for example, an individual could over-perform or under-perform at an assessment and be classified in a group that is not characteristic of their state. Misclassification was used to account for measurement error in the cognitive testing, where the observed states are treated as misclassified observations of the underlying latent states [11]. State misclassification is allowed to occur at any interview but the probabilities are independent across individuals. It is possible to assess covariate effects on the misclassification rates as well as on the cognitive transitions. However, as they are mutually dependent processes within the likelihood, the inclu- sion of cognitive lifestyle as a covariate on both would result in an over-fitted model. As the primary interest of this study is to examine the effects of cognitive lifestyle on cognitive decline, cognitive lifestyle was not added as a covariate on the misclassification rates as decline and misclassification are dependent processes in the model. We have therefore assumed a null effect of cognitive lifestyle on misclassification. The model was specified so that the initial state probabilities were estimated from the data taking into account misclassification. Three covariates were included in the analysis: age, gender, and a measure of cognitive lifestyle. Age was entered as a time dependent covariate. The Broyden-Fletcher-Goldfarb-Shanno (BFGS) optimization method was used to maximize the likelihood for the multi-state models. Robustness of parameter estimates was tested by running the models from two different sets of starting values. All data were analyzed using $\mathrm{R}$ version 2.12.2 [12]; the multistate model was formulated and fitted using the 'msm' package [13].

\section{RESULTS}

The distribution of age, gender, cognitive lifestyle score, and initial cognitive group by study centre are presented in Table 1. Age and gender distribution are similar across the five centers (mean age 75 years, $40 \%$ men). Cognitive states at baseline were also similarly distributed across the centers with around 50\% having no impairment, $30 \%$ with slight impairment, $15 \%$ with moderate impairment, and $5 \%$ with severe impairment. The maximum number of observations for a single individual was ten; all of the state transitions are shown in Table 2.

Table 1

Summary of population characteristics at baseline

\begin{tabular}{|c|c|c|c|c|c|c|}
\hline & \multicolumn{6}{|c|}{ Study center } \\
\hline & Cambs* & Gwynedd & Newcastle & Nottingham & Oxford & Overall \\
\hline Age (s.d.) & $75.0(7.1)$ & $75.5(6.7)$ & $75.0(6.8)$ & $75.1(6.8)$ & $75.4(6.9)$ & $75.2(6.9)$ \\
\hline \multicolumn{7}{|l|}{ Gender $-n(\%)$} \\
\hline Male & $1,090(43.3)$ & $1,037(41.2)$ & $899(37.0)$ & $980(40.7)$ & $1,026(39.1)$ & $5,032(40.3)$ \\
\hline Female & $1,428(56.7)$ & $1,478(58.8)$ & $1,532(63.0)$ & $1,426(59.3)$ & $1,596(60.9)$ & $7,460(59.7)$ \\
\hline \multicolumn{7}{|l|}{ Cognitive lifestyle score $-n(\%)$} \\
\hline Tertile 1 (Low) & $919(36.5)$ & $656(26.1)$ & $732(30.1)$ & $894(37.2)$ & $868(33.1)$ & $4,069(32.6)$ \\
\hline Tertile 2 (Medium) & $765(30.4)$ & $668(26.6)$ & $820(33.7)$ & 888 (36.9) & $743(28.3)$ & $3,884(31.1)$ \\
\hline Tertile 3 (High) & $834(33.1)$ & $1,191(47.4)$ & $879(36.2)$ & $624(25.9)$ & $1,011(38.6)$ & $4,539(36.3)$ \\
\hline \multicolumn{7}{|l|}{ MMSE group $-n(\%)$} \\
\hline Normal ability (27-30) & $1,211(48.1)$ & $1,315(52.3)$ & $1,345(55.3)$ & $1,401(58.2)$ & $1,329(50.7)$ & $6,601(52.8)$ \\
\hline Slight impairment (23-26) & 829 (32.9) & $791(31.5)$ & $748(30.8)$ & $659(27.4)$ & $855(32.6)$ & $3,882(31.1)$ \\
\hline Moderate impairment (18-22) & 370 (14.7) & 294 (11.7) & $246(10.1)$ & $247(10.3)$ & $339(12.9)$ & $1,496(11.9)$ \\
\hline Severe impairment $(<18)$ & $108(4.3)$ & $115(4.6)$ & $92(3.8)$ & $99(4.1)$ & $99(3.8)$ & $513(4.1)$ \\
\hline
\end{tabular}


Table 2

Summary of all state transitions

\begin{tabular}{|c|c|c|c|c|c|c|}
\hline & \multicolumn{6}{|c|}{ MMSE scores } \\
\hline \multicolumn{7}{|c|}{ To } \\
\hline From & $<18$ & $18-22$ & $23-26$ & $27-30$ & Death & Censored \\
\hline $27-30$ & $96(<1 \%)$ & $351(2 \%)$ & $2,517(16 \%)$ & $7,174(46 \%)$ & $3,617(23 \%)$ & $1,956(12 \%)$ \\
\hline $23-26$ & $259(3 \%)$ & $1,146(11 \%)$ & $2,877(29 \%)$ & $1,837(18 \%)$ & $2,953(30 \%)$ & $929(9 \%)$ \\
\hline $18-22$ & $580(13 \%)$ & $1,164(27 \%)$ & $686(16 \%)$ & $98(2 \%)$ & $1,545(36 \%)$ & $256(6 \%)$ \\
\hline$<18$ & $910(39 \%)$ & $172(7 \%)$ & $39(2 \%)$ & $1(<1 \%)$ & $1,188(50 \%)$ & $48(2 \%)$ \\
\hline
\end{tabular}

Table 3

Hazard ratios of age, gender, and cognitive lifestyle score upon cognitive transitions

\begin{tabular}{|c|c|c|c|c|}
\hline \multirow[b]{2}{*}{ Covariate - transition } & \multicolumn{4}{|c|}{ Hazard ratio ( $95 \%$ confidence interval) } \\
\hline & Age (years) & Gender (Women) & $\begin{array}{c}\text { Cognitive lifestyle } \\
\text { (Medium versus Low) }\end{array}$ & $\begin{array}{l}\text { Cognitive lifestyle } \\
\text { (High versus Low) }\end{array}$ \\
\hline State 1 - State 2 & $1.14(1.12,1.16)$ & $1.21(0.99,1.48)$ & $0.92(0.72,1.18)$ & $0.58(0.45,0.74)$ \\
\hline State 1 - Death & $1.05(1.04,1.06)$ & $0.55(0.47,0.64)$ & $0.88(0.71,1.09)$ & $0.83(0.69,1.00)$ \\
\hline State 2 - State 1 & $0.76(0.68,0.85)$ & $0.54(0.30,0.98)$ & $0.74(0.26,2.13)$ & $2.93(1.35,6.38)$ \\
\hline State 2 - State 3 & $1.09(1.08,1.11)$ & $1.10(0.90,1.34)$ & $0.73(0.58,0.92)$ & $0.81(0.65,1.01)$ \\
\hline State 2 - Death & $1.06(1.05,1.08)$ & $0.55(0.45,0.68)$ & $1.27(0.99,1.64)$ & $0.90(0.66,1.24)$ \\
\hline State 3 - State 2 & $0.88(0.81,0.95)$ & $0.42(0.18,0.98)$ & $1.79(0.59,5.43)$ & $2.28(0.83,6.30)$ \\
\hline State 3 - State 4 & $1.08(1.07,1.09)$ & $0.94(0.76,1.16)$ & $1.15(0.94,1.40)$ & $1.08(0.87,1.34)$ \\
\hline State 3 - Death & $1.03(1.00,1.05)$ & $0.41(0.30,0.56)$ & $0.65(0.41,1.04)$ & $1.13(0.78,1.65)$ \\
\hline State 4 - Death & $1.03(1.03,1.04)$ & $0.80(0.72,0.91)$ & $1.19(1.06,1.33)$ & $1.28(1.12,1.45)$ \\
\hline
\end{tabular}

State 1 (no impairment): MMSE 27-30, State 2 (slight impairment): MMSE 23-26, State 3 (moderate impairment): MMSE 18-22, State 4 (severe impairment): MMSE 0-17.

The initial state occupancies estimated from the multi-state model were $49 \%$ no impairment, $33 \%$ for slight impairment, $14 \%$ for moderate impairment, and $4 \%$ for severe impairment. The observed proportions were $53 \%, 31 \%, 12 \%$, and $4 \%$ (differences are due to the misclassification).

The hazard ratios of the covariate effects on the transitions are shown in Table 3. Aging was found to associate with an increased risk of moving to a more impaired cognitive state. Hazard ratios per additional year of life varied from 1.08 to 1.14 , which represent an $8-14 \%$ increased risk per year of life. Younger persons were more likely to back transition from slight impairment to no impairment (hazard ratio (HR), 95\% Confidence Interval $-0.76(0.68,0.85))$ and from moderate to slight impairment $(0.88(0.81,0.95))$. There were differences in the transitions by gender. Females had a reduced rate of moving from any cognitive state to death (HRs varied from 0.41 to 0.80 ). They were also less likely to transition back from a slightly impaired state to a non-impaired state $(0.54(0.30,0.98))$.

The hazard ratios for the primary variable of interest, cognitive lifestyle score, showed significant differences between those in the upper versus the lower tertile with higher cognitive lifestyle associating with a reduced risk of moving from no impairment to slight impairment - HR 0.58, 95\% CI (0.45, 0.74). A higher cognitive lifestyle score was associated with an increased chance of moving back from slight impairment to no impairment -2.93 (1.35, 6.38). Finally, a higher cognitive lifestyle score was associated with an increased mortality risk from within the severely impaired cognitive state $-1.28(1.12,1.45)$. A slightly weaker version of the same finding was observed in those who were in the middle tertile for cognitive lifestyle score $-1.19(1.06,1.33)$. The transitions and hazard ratios for the highest versus lowest tertile of cognitive lifestyle score are shown in Fig. 1.

The estimated misclassification showed that the probability of incorrect classification was most common in the slightly $(33 \%)$ and moderately impaired groups $(34 \%)$. The true cognitive states of those individuals incorrectly classified by the model were likely to be more impaired than was actually observed. Of those incorrectly classified from the slightly impaired state, the chance of being observed as having no impairment was $82 \%$. Similarly, of those incorrectly classified from the moderately impaired state, the chance of being observed in a slightly impaired state was also $82 \%$. Misclassification was much lower in the non-impaired (11\%) and severely impaired categories $(13 \%)$ 


\section{DISCUSSION}

This large, multi-center population-based study of older persons from across England and Wales reveals significant associations between cognitive lifestyle score and cognitive function. Hazard ratios comparing the highest versus lowest tertile of cognitive lifestyle score showed low CLS to associate with an increased risk of moving from a high MMSE score (27-30) to a lower MMSE score (23-26). High CLS was associated with a positive transition from the slightly impaired MMSE state (23-26) to the non-impaired state but also with an accelerated risk of death from severe impairment $(\mathrm{MMSE}<18)$.

These results are supportive of the reserve hypothesis proposed by Stern [3, 4] with greater education, socioeconomic status, and social engagement in oldage protecting against cognitive decline. Despite being slightly counter-intuitive, higher mortality in those with higher cognitive lifestyle scores also fits well within the proposed pathway. While it takes longer for those with higher cognitive lifestyle scores to reach a severely impaired state, once this happens they are more likely to have a faster transition to death than those with low cognitive lifestyle scores. This implies that someone with a high cognitive lifestyle score is better able to compensate for initial degenerative brain changes, however, when cognitive impairment becomes obvious, the underlying brain damage is so severe that a fast transition to death is more likely than a prolonged clinical course. However, this could occur via different pathways. For example, the slope of decline may be equal for all but those with higher reserve have higher initial cognition and hence reach a severely impaired state later in life and having declined more than those with low reserve. This observation may also be due to increased brain (and not cognitive) reserve. More educated individuals may have larger brains or more cortical tissue, which protects them from expressing clinical signs of decline compared to those with low education. Support for this claim comes from a recent neuropathology paper that included the CFAS cohort found increased education to associate with increased brain weight (odds ratio 1.14 [1.06, 1.24]) [14]. Finally, assuming that those with higher reserve again achieve greater scores, they might decline at an older age but at a faster rate than those with low reserve.

Such pathways have been alluded to by Reuser et al. [15], who found increased education to delay the onset of cognitive impairment but increase the mortality risk from an impaired state. Furthermore, Wilson and colleagues [16] showed increased cognitive activity, as measured by activities such as listening to the radio, reading newspapers, completing puzzles, etc., to be associated with reduced cognitive decline prior to dementia onset but accelerated decline thereafter. A recent analysis on MRC CFAS data found high CLS to have a protective effect against incident dementia and a negative but not statistically significant effect on survival time after dementia diagnosis. The hazard ratio for survival time after dementia diagnosis for those with a high CLS was $1.395 \%$ CI $(1.0,1.7)$ [10]. The present study went beyond a dementia-non-dementia analysis to examine cognitive aging across all levels of cognitive ability.

Our findings also suggested high CLS is associated with an increased chance of moving from a slightly impaired state back to a non-impaired state. The effect size was considerably larger for CLS compared to young age, which also increased the chance of cognitive recovery. This may be due to MMSE measurement error with non-impaired individuals performing poorly at one interview. However, upon examining state misclassification, we found that the fitted model categorized $27 \%$ of those observed in the slightly impaired state as non-impaired. This reduces the possibility of the finding being explained by fluctuations in MMSE scores. It is possible that within the MMSE groupings individuals with a low CLS had lower MMSE scores making them more likely to change group if they dropped a single point between waves. However, this is difficult to examine due to the complex study design of CFAS; individuals attended a different number of waves and at different times. Thus any MMSE means by CLS status for the four MMSE groups would be biased by individuals who were seen on a more regular basis. In addition, a baseline analysis of raw MMSE scores by group (where all participants were assessed) would provide limited information as the analyses and covariate effects were assessed over all waves and data points.

There were several strengths of this investigation, including 44,891 state measurements on a populationrepresentative sample of 12,492 older persons across England and Wales; there were up to ten cognitive measurements on a single individual over a 16 year period. The use of state-based modeling yielded an easily interpretable model that was able to accommodate potential state misclassification, death, and censoring. Furthermore, many previous studies of cognitive lifestyle and cognition have focused on small samples with AD/dementia or MCI [17-19] as opposed to a heterogeneous population-based sample that encompasses all 
levels of cognitive ability. Furthermore, whilst there were statistically significant differences in the hazards of cognitive transitions for men and women, a sensitivity analysis found no evidence for an interaction between gender and cognitive lifestyle (results not shown).

The specific aim we set out to investigate in this paper was whether cognitive lifestyle was associated with all stages of late-life cognitive decline, cognitive recovery, and mortality risk from each cognitive state. Whether modification of cognitive lifestyle in late-life affects subsequent cognitive change by delaying the onset of decline and death or improving cognitive function is not testable within the current model. A further limitation lies in our assumption that CLS has no effect on misclassification. However, the empirical testing of this assumption is complex as any effects of CLS on misclassification may be due to the effects of CLS on cognitive decline or vice versa. Nonetheless, prior to the analyses presented in this paper, we ran a model of CLS on cognitive decline without misclassification and found similar results (not shown). The addition of state misclassification adds a further layer of complexity to our analysis but enables a more realistic model of cognitive decline.

Other limitations include the definition and potential misclassification of cognitive states, particularly the slight/moderate impairment groups. We attempted to account for incorrect categorizations by including misclassification in our model. Although widely used as a diagnostic entity of sub-clinical cognitive impairment [20], there are many different definitions of MCI [21]. However, using MMSE scores to predict future dementia in population representative samples is as accurate as other methods that use more complex assessments of cognition [9, 22]. It is known that MMSE scores are influenced by age and education [23]. However, in our model the covariate effects on the multi-state model transitions are conditional on the previous cognitive state, including baseline cognitive state for the first transition. Furthermore, education and age (time-dependent variable) are also covaried for in the model. This nullifies the age/education bias that may be present when analyzing MMSE scores univariately. Future models will also need to explore the impact of other covariates, such as depression, cardiovascular disease risk factors, and APOE4, on the cognitive transitions and how they interact with the cognitive lifestyle variables. However, this will be computationally intensive and may lead to identifiability problems in the estimation of covariates effects. Dealing with these problems requires methodological development from the multi-state modeling framework applied in this paper.

Finally, it is unclear how assignment to states and trajectories based on cognitive test data compares with the changes seen in the brain. One of the most notable challenges in understanding the neurobiology of aging is the discrimination of normal brain aging from neural pathologies [24]. To investigate this properly requires neuropathology identified in the brain after death. However, most cognitive healthy individuals display a varying degree of neuropathological features that are typical of $\mathrm{AD}$ [25]. It is uncertain how cognitive lifestyle factors interact with both the development and impact of neural pathologies. A recent analysis of 872 older persons ( $>65$ years) from three studies across Europe, including MRC CFAS, found education was not associated with neuropathological burden although it was associated with a reduced risk of developing dementia [14]. This ties in with a compensation mechanism as opposed to neuroprotection. A study on 66 healthy elderly controls and 17 persons with AD found amyloid deposits led to lower cognitive performance in both groups [18]. However, adjustment for cognitive reserve (measured by education and a vocabulary-based test of pre-morbid cognitive ability - the National Adult Reading Test) attenuated the findings, leading the authors to conclude that reserve may have a protective effect against amyloid-related cognitive impairment.

In conclusion, there is evidence to associate an active cognitive lifestyle with a decreased rate of cognitive decline, an increased chance of cognitive 'recovery' but also an increased mortality risk from a severely impaired state. The latter finding may indicate a compression of morbidity for those with an active cognitive lifestyle. Future studies should aim to incorporate neuropathology into longitudinal studies of cognitive change to better understand the biological basis for these 'reserve' effects and determine the relative importance of each cognitive lifestyle factor in predicting cognitive decline.

\section{ACKNOWLEDGMENTS}

This work was supported by a Medical Research Council project grant (MRC G9901400). Fiona Matthews and Ardo van den Hout are funded under MRC programme grant UC_US_A030_0031. Riccardo Marioni is an Alzheimer's Research UK Fellow (ART-RF2010-2). Michael Valenzuela is a University of New South Wales Vice Chancellor's Research 
Fellow. He is supported by National Health and Medical Research Council of Australia (NHMRC) project grant (ID 630551) and is funded by a NHMRC Clinical Career Development Award. The study is part of the Cambridge and Peterborough CLAHRC.

Authors' disclosures available online (http://www.jalz.com/disclosures/view.php?id=990).

\section{REFERENCES}

[1] Valenzuela MJ, Sachdev P (2006) Brain reserve and cognitive decline: A non-parametric systematic review. Psychol Med 36, 1065-1073.

[2] Valenzuela MJ, Sachdev P (2006) Brain reserve and dementia: A systematic review. Psychol Med 36, 441-454.

[3] Stern Y (2002) What is cognitive reserve? Theory and research application of the reserve concept. J Int Neuropsychol Soc 8, 448-460.

[4] Stern Y (2009) Cognitive reserve. Neuropsychologia 47, 2015-2028.

[5] Stern Y, Tang MX, Denaro J, Mayeux R (1995) Increased risk of mortality in Alzheimer's disease patients with more advanced educational and occupational attainment. Ann Neurol 37, 590-595.

[6] Xie J, Brayne C, Matthews FE (2008) Survival times in people with dementia: Analysis from population based cohort study with 14 year follow-up. BMJ 336, 258-262.

[7] MRC CFAS (1998) Cognitive function and dementia in six areas of England and Wales: The distribution of MMSE and prevalence of GMS organicity level in the MRC CFA Study. Psychol Med 28, 319-335.

[8] Folstein MF, Folstein SE, McHugh PR (1975) "Mini-mental state". A practical method for grading the cognitive state of patients for the clinician. J Psychiatr Res 12, 189-198.

[9] Stephan BCM, Savva GM, Brayne C, Bond J, McKeith IG, Matthews FE, MRC CFAS (2010) Optimizing mild cognitive impairment for discriminating dementia risk in the general older population. Am J Geriatr Psychiatry 18, 662-673.

[10] Valenzuela MJ, Brayne C, Sachdev P, Wilcock G, Matthews FE, MRC CFAS (2010) Cognitive lifestyle and long-term risk of dementia and survival after diagnosis in a multicentre population-based cohort. Am J Epidemiol 173, 1004-1012.

[11] van den Hout A, Matthews FE (2008) Multi-state analysis of cognitive ability data: A piecewise-constant model and a Weibull model. Stat Med 27, 5440-5455.
[12] R Development Core Team: R, A Language and Environment for Statistical Computing. R Foundation for Statistical Computing. Vienna, Austria; 2010 (ISBN) 3-900051-07-0, http://www. R-project.org.

[13] Jackson CH, Sharples LD, Thompson SG, Duffy SW, Couto E (2003) Multistate Markov models for disease progression with classification error. The Statistician 52, 193-209.

[14] EClipSE (2010) Education, the brain and dementia: Neuroprotection or compensation? Brain 133, 2210-2216.

[15] Reuser M, Willekens FJ, Bonneux L (2011) Higher education delays and shortens cognitive impairment. A multistate life table analysis of the US Health and Retirement Study. Eur J Epidemiol 26, 395-403.

[16] Wilson RS, Barnes LL, Aggarwal NT, Boyle PA, Hebert LE, Mendes de Leon CF, Evans DA (2010) Cognitive activity and the cognitive morbidity of Alzheimer disease. Neurology $\mathbf{7 5}$, 990-996.

[17] Del Ser T, Hachinski V, Merskey H, Munoz DG (1999) An autopsy-verified study of the effect of education on degenerative dementia. Brain 122(Pt 12), 2309-2319.

[18] Rentz DM, Locascio JJ, Becker JA, Moran EK, Eng E, Buckner RL, Sperling RA, Johnson KA (2010) Cognition, reserve, and amyloid deposition in normal aging. Ann Neurol 67, 353364.

[19] Rolstad S, Nordlund A, Eckerstrom C, Gustavsson MH, Blennow K, Olesen PJ, Zetterberg H, Wallin A (2010) High education may offer protection against tauopathy in patients with mild cognitive impairment. J Alzheimers Dis 21, 221228.

[20] Davis HS, Rockwood K (2004) Conceptualization of mild cognitive impairment: A review. Int J Geriatr Psychiatry 19, 313-319.

[21] Stephan BC, Brayne C, McKeith IG, Bond J, Matthews FE (2008) Mild cognitive impairment in the older population: Who is missed and does it matter? Int J Geriatr Psychiatry 23, 863-871.

[22] Busse A, Bischkopf J, Riedel-Heller SG, Angermeyer MC (2003) Mild cognitive impairment: Prevalence and predictive validity according to current approaches. Acta Neurol Scand 108, 71-81.

[23] Crum RM, Anthony JC, Bassett SS, Folstein MF (1993) Population-based norms for the Mini-Mental State Examination by age and educational level. JAMA 269, 2386-2391.

[24] Whalley LJ (2002) Brain ageing and dementia: What makes the difference? Br J Psychiatry 181, 369-371.

[25] MRC CFAS (2001) Pathological correlates of late-onset dementia in a multicentre, community-based population in England and Wales. Lancet 357, 169-175. 